Cite this: Chem. Commun., 2011, 47, 1707-1709

\section{A Covalent Organic Framework with 4 nm open pores $\dagger$}

\author{
Mirjam Dogru, ${ }^{a}$ Andreas Sonnauer, ${ }^{a}$ Andrei Gavryushin, ${ }^{b}$ Paul Knochel ${ }^{b}$ and Thomas Bein $* a$ \\ Received 10th September 2010, Accepted 18th November 2010 \\ DOI: $10.1039 / \mathbf{c 0 c 0 3 7 9 2 c}$
}

The synthesis and characterization of a new mesoporous Covalent Organic Framework BTP-COF is described, the latter having fully accessible pores with an open diameter of $4.0 \mathrm{~nm}$.

Porous crystalline materials with a well-defined pore size distribution are essential for applications in gas storage, ${ }^{1}$ catalysis, separation, ${ }^{2}$ optics ${ }^{3}$ and chemical sensing. ${ }^{4}$ Specifically, large pores reaching several $\mathrm{nm}$ diameter permit the design of a multitude of nano-devices interacting with large guest molecules. ${ }^{5}$

To gain maximum control over local structure, chemistry and physics in the pore system, it would be highly desirable to have access to multi-nm pores with molecularly defined walls. ${ }^{6}$ For example, Sonnauer et al. reported a mesoporous Metal-Organic Framework (MOF) exhibiting giant cages with diameters of up to $4.6 \mathrm{~nm}$, although the windows connecting these cages are much smaller with dimensions of about $2.0 \mathrm{~nm}^{7}$ Other mesoporous MOFs with similar pore dimensions have been described. ${ }^{8}$ A new class of materials called Covalent Organic Frameworks (COFs) ${ }^{9}$ with organic open pore structures exhibit significant stability due to covalent bonding. ${ }^{10}$ They feature great structural diversity and also allow precise geometric control through the design of the molecular building blocks (reticular chemistry). ${ }^{11}$ Until now mesoporous COFs with pore sizes larger than $3.4 \mathrm{~nm}$ have not been reported. ${ }^{12}$

Here we describe the synthesis and characterization of a new mesoporous Covalent Organic Framework BTP-COF, with fully accessible pores having an open diameter of $4.0 \mathrm{~nm}$. To the best of our knowledge, this is the largest open pore diameter of any crystalline material ever reported. The new BTP-COF was synthesized under solvothermal conditions by co-condensation of 1,3,5-benzenetris(4-phenylboronic acid) (BTPA) and the polyol 2,3,6,7-tetrahydroxy-9,10-dimethyl-anthracene (THDMA) (Fig. 1). The synthesis parameters for the new COF structure were

\footnotetext{
${ }^{a}$ Department of Chemistry and Center for NanoScience (CeNS), University of Munich (LMU), Butenandtstr. 5-13 (E),

81377 Munich, Germany.E-mail: bein@lmu.de;

Fax: + 49 (0)89-2180-77622; Tel: + 49 (0)89-2180-77623

${ }^{b}$ Department of Chemistry, University of Munich,

Butenandtstr. 5-13 (F), 81377 Munich, Germany.

E-mail: knoch@cup.uni-muenchen.de, +49(0)89-2180-77680;

Tel: + 49 (0)89-2180-77681

$\dagger$ Electronic supplementary information (ESI) available: Synthesis optimization of BTP-COF with high throughput methods, characterization of BTP-COF with structural models and X-ray analyses, FT-IR spectroscopy, ss-NMR, SEM and TGA data. CCDC 796269. For ESI and crystallographic data in CIF or other electronic format see DOI: $10.1039 / \mathrm{c} 0 \mathrm{cc} 03792 \mathrm{c}$
}

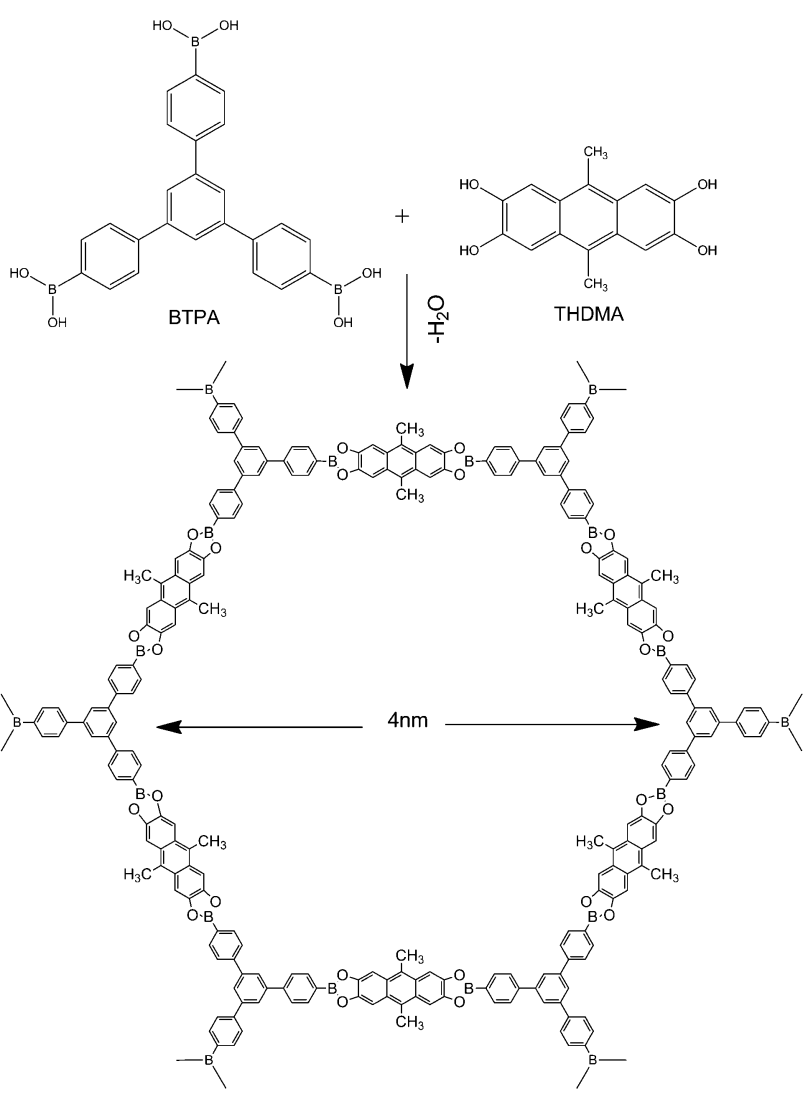

Fig. 1 Co-condensation reaction for the BTP-COF with $4.0 \mathrm{~nm}$ pores.

found and optimized in a high throughput screening approach using a robotic dosing system. ${ }^{13}$

The investigated reaction parameters were solvents, solvent ratio, concentration, ratio of the starting materials, time and temperature. Thereby it was observed that the solvent mixture is the most important parameter for successful network formation, whereas the latter ones influence the purity (crystallinity and yield) of the system. We can conclude that the reversibility of the ester condensation depends strongly on the polarity of the reaction solution. Hence the crystallization is only possible if the appropriate reaction conditions are adapted for every linker system. The COF materials were obtained under microwave synthesis conditions. ${ }^{14}$ The different reaction parameters are described in the ESI. $\dagger$

Powder X-ray diffraction (PXRD) confirms the formation of a highly crystalline Covalent Organic Framework. In order 


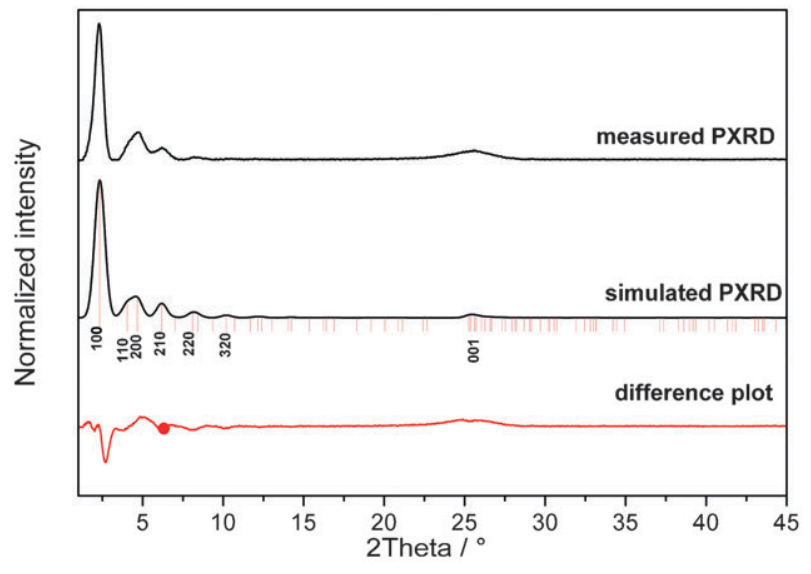

Fig. 2 The experimentally observed PXRD pattern, after degassing the sample at $150{ }^{\circ} \mathrm{C}$ for $2 \mathrm{~d}$, the simulated pattern and the difference plot in red.

to identify the new phase, possible structures were modelled with MS Studio. ${ }^{15}$ Based on the calculated structures, the corresponding powder patterns were simulated and compared to the experimentally obtained data. As reported for previous COF structures, the potential stacking types of the hexagonal planar sheets were calculated. ${ }^{9}$ In principle two distinct arrangements are viewed as most likely.

The adjacent sheets can stack in an eclipsed way (AA type), resulting in accessible pores in one dimension (along the $c$-axis). Alternatively, the adjacent sheets can form a staggered array (AB type) as in graphite (see ESI $\dagger$ ). The experimental PXRD pattern for BTP-COF agrees very well with the simulated pattern for an eclipsed AA arrangement (Fig. 2). The eclipsed BTP-COF has a hexagonal P6/mmm symmetry. Unit cell parameters determined from the experimental X-ray patterns match very well those obtained from the structure simulations (peak broadening included).

The (001) reflection is broadened, which can be attributed to disorder in the spacing and/or to a small domain size along this axis. The measured data were compared to several simulated structures. The best fit was obtained for an interlayer distance of $0.352 \mathrm{~nm}$ (see Fig. S1, ESI $\dagger$ ), which is larger than the

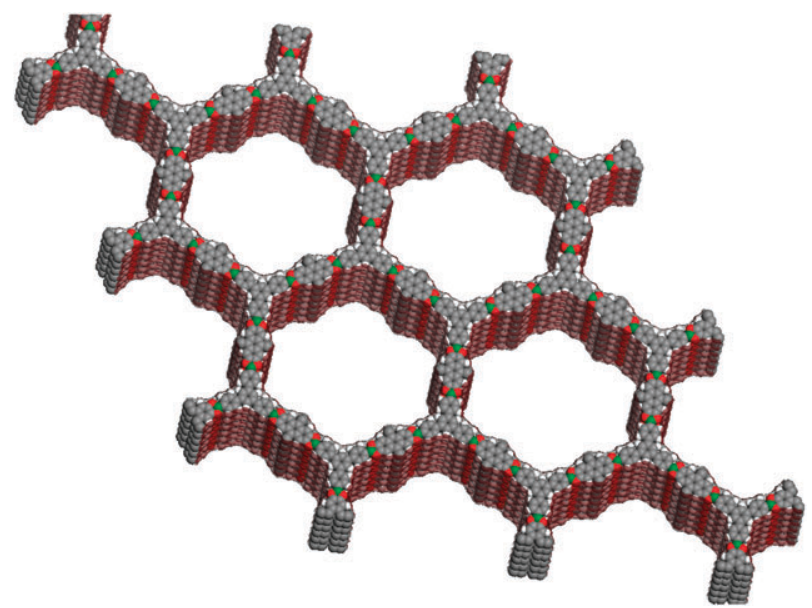

Fig. 3 Schematic structure of BTP-COF including the Connolly surface, based on calculations using the universal force field method and crystal lattice parameters (boron in green, oxygen in red and carbon in grey).
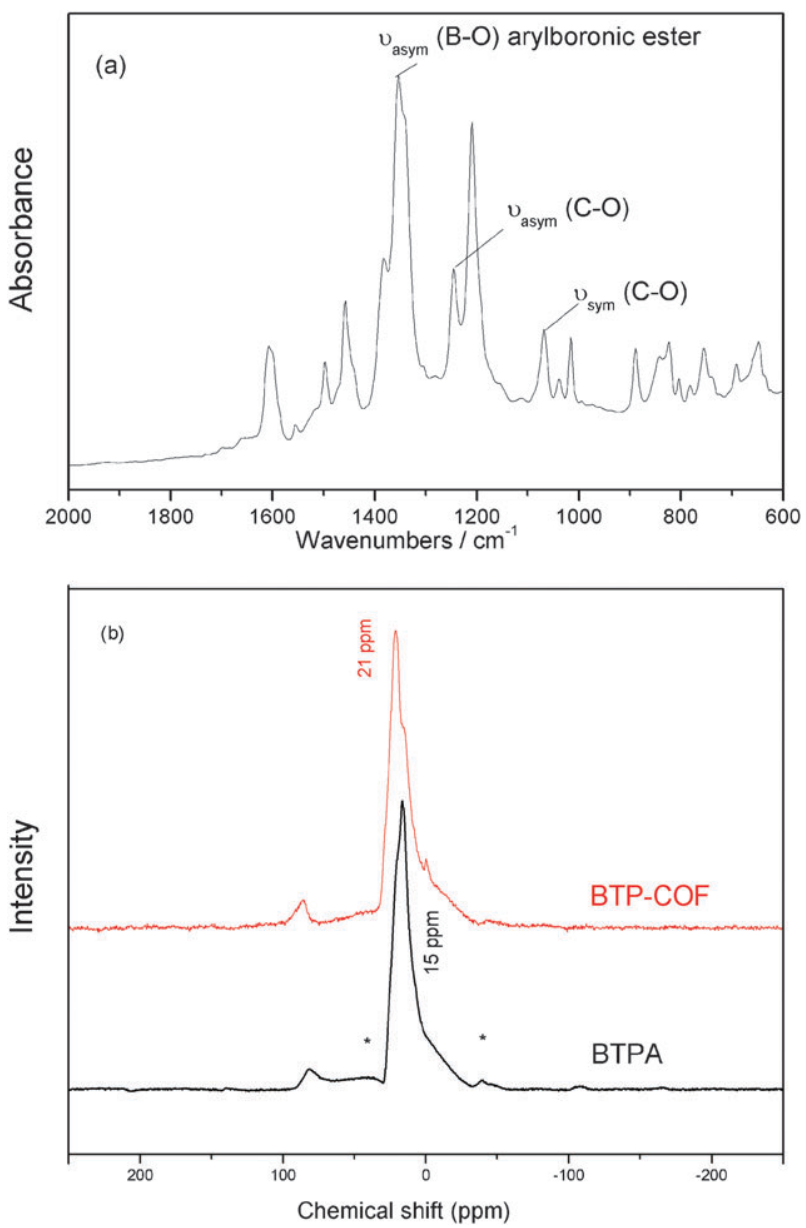

Fig. 4 (a) FTIR spectrum of BTP-COF, showing the characteristic aryl boronate ester functionality, (b) ${ }^{11} \mathrm{~B}$ MAS NMR spectrum of BTP-COF.

spacing of $0.346 \mathrm{~nm}$ in $\mathrm{COF}-5,{ }^{9}$ a structure related to that of boron nitride. ${ }^{16}$ The slightly larger distance between the sheets along the $c$-axis can be attributed to the steric requirements of the methyl groups in the pores.

The resulting structure of the new Covalent Organic Framework is shown in Fig. 3. Based on the structure, we can also draw conclusions about the expected pore filling of the framework (see sorption data below). ${ }^{17}$

The presence of the building blocks in the assembly was confirmed with FTIR spectroscopy (Fig. 4a). Consistent with previously reported COF-materials, the attenuation of the $\mathrm{OH}$-stretching band indicates esterification of the boronic acids with the polyols. The bands at $1386 \mathrm{~cm}^{-1}$ and $1356 \mathrm{~cm}^{-1}$ indicate that the expected boronate ester functionality is present. The ${ }^{11} \mathrm{~B}$ MAS NMR spectrum (Fig. 4b) shows the formation of tricoordinated planar boron with a signal at $21 \mathrm{ppm}$ which differs from the starting material, the BTPA (15 ppm).

Thermogravimetric analysis indicates that BTP-COF is stable up to $400{ }^{\circ} \mathrm{C}$. Heating to $220{ }^{\circ} \mathrm{C}$ is accompanied by a mass loss of $12 \%$, which can be attributed to volatile guest molecules in the mesopores. At $600{ }^{\circ} \mathrm{C}$ a total weight loss of $84 \%$ is reached. The remaining $16 \%$ correspond to the stoichiometrically expected amount of $\mathrm{B}_{2} \mathrm{O}_{3}$. In order to remove guest molecules from the pores, the crude product of 

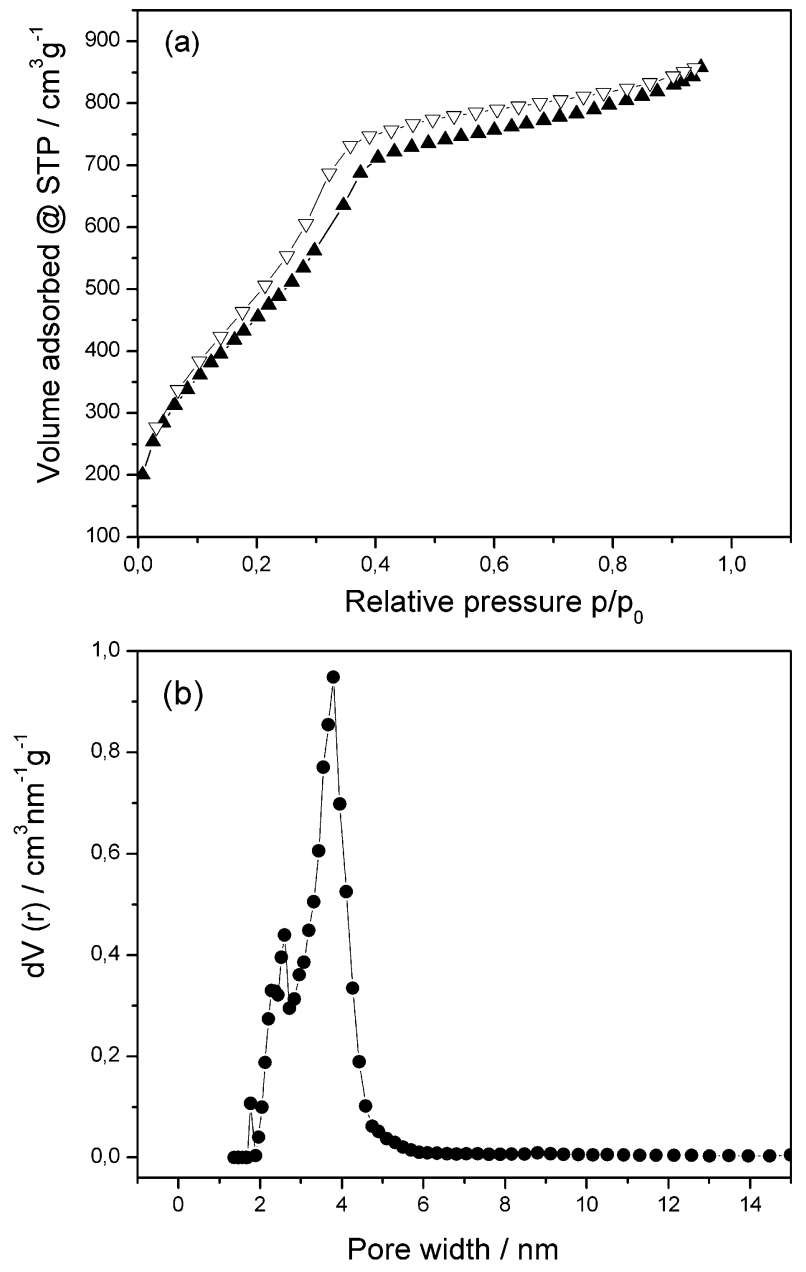

Fig. 5 (a) Nitrogen sorption isotherm of degassed BTP-COF measured at $-196{ }^{\circ} \mathrm{C}$ and (b) pore size distribution.

BTP-COF was soaked in acetone for $2 \mathrm{~d}$ and subsequently dried at $150{ }^{\circ} \mathrm{C}$ in vacuum for at least $12 \mathrm{~h}$. As shown in the powder pattern of BTP-COF after removal of the guest molecules (Fig. 2), there is no collapse of the crystalline structure and the stacking of the layers is preserved. Furthermore, BTP-COF is stable for several months in air, and it does not degrade in most of the common organic solvents.

The porosity of BTP-COF was confirmed by $\mathrm{N}_{2}$-sorption measurements after degassing of the sample at elevated temperature. The isotherm was recorded at $-196{ }^{\circ} \mathrm{C}$. BTP-COF exhibits a type IV isotherm (Fig. 5a) characteristic for mesoporous materials, ${ }^{18}$ and a very high BrunauerEmmett-Teller (BET) surface area of almost $2000 \mathrm{~m}^{2} \mathrm{~g}^{-1}$. The pore size distribution of BTP-COF shows pores with a pore diameter of around $4.0 \mathrm{~nm}$ (Fig. $5 \mathrm{~b}$ ).

In order to assess whether the pores in the sample are accessible or blocked by parts of the framework or solvent molecules, the surface area of crystalline materials can be predicted from the crystal structure using geometric methods. ${ }^{19}$ The Connolly surface is calculated by simulating a probe molecule (in this case nitrogen) rolling along the surface. ${ }^{20}$ Another method to calculate the surface and pore volume is the accessible surface area. ${ }^{21}$
Table 1 Theoretical and experimental values for surface area and pore volume of BTP-COF

\begin{tabular}{lll}
\hline & Surface area $/ \mathrm{m}^{2} \mathrm{~g}^{-1}$ & Volume $/ \mathrm{cm}^{3} \mathrm{~g}^{-1}$ \\
\hline Connolly surface & 2000 & 1.63 \\
Access. surface & 1764 & 1.29 \\
Sorption data & 1850 & 1.29 \\
\hline
\end{tabular}

The value for the BET surface area calculated from experimental nitrogen adsorption isotherms is in good accordance with the theoretically calculated values (Table 1).

As shown above, we have synthesized a new mesoporous crystalline material with extremely large permanent pores using the concept of reticular chemistry. For the first time a crystalline open framework structure with accessible $4.0 \mathrm{~nm}$ pores is reported. We anticipate this structure and related materials to offer an attractive platform for the covalent attachment of large molecular functional guests, thus creating a highly controlled environment and well-defined interactions between the crystalline walls of the host and the guest species.

\section{Notes and references}

1 (a) J.-R. Li, R. J. Kuppler and H.-C. Zhou, Chem. Soc. Rev., 2009, 38, 1477; (b) H. Furukawa and O. M. Yaghi, J. Am. Chem. Soc., 2009, 131, 8875.

2 J. S. Seo, D. Whang, H. Lee, S. I. Jun, J. Oh, Y. J. Jeon and K. Kim, Nature, 2000, 404, 982.

3 J. Kobler, B. V. Lotsch, G. A. Ozin and T. Bein, ACS Nano, 2009, 3, 1669.

4 F. Schüth, K. Sing and J. Weitkamp, Handbook of Porous Solids, Wiley-VCH, Weinheim, 2008.

5 G. Ferey, C. Mellot-Draznieks, C. Serre, F. Millange, J. Dutour, S. Surble and I. Margiolaki, Science, 2005, 309, 2040.

6 G. Férey, C. Serre, C. Mellot-Draznieks, F. Millange, S. Surblé, J. Dutour and I. Margiolaki, Angew. Chem., 2004, 116, 6456.

7 A. Sonnauer, F. Hoffmann, M. Fröba, L. Kienle, V. Duppel, M. Thommes, C. Serre, G. Férey and N. Stock, Angew. Chem., 2009, 121, 3849.

8 (a) N. Klein, I. Senkovska, K. Gedrich, U. Stoeck, A. Henschel, U. Mueller and S. Kaskel, Angew. Chem., 2009, 121, 10139; (b) Y. Park, et al., Angew. Chem., 2007, 119, 8237.

9 A. P. Côté, A. I. Benin, N. W. Ockwig, M. O'Keeffe, A. J. Matzger and O. M. Yaghi, Science, 2005, 310, 1166.

10 (a) G. Couderc and J. Hulliger, Chem. Soc. Rev., 2010, 39, 1545; (b) R. W. Tilford, W. R. Gemmill, H.-C. zur Loye and J. J. Lavigne, Chem. Mater., 2006, 18, 5296.

11 O. M. Yaghi, M. O'Keeffe, N. W. Ockwig, H. K. Chae, M. Eddaoudi and J. Kim, Nature, 2003, 423, 705.

12 (a) A. P. Côté, H. M. El-Kaderi, H. Furukawa, J. R. Hunt and O. M. Yaghi, J. Am. Chem. Soc., 2007, 129, 12914; (b) S. Wan, J. Guo, J. Kim, H. Ihee and D. Jiang, Angew. Chem., 2009, 121, 3253; (c) C. J. Doonan, D. J. Tranchemontagne, T. G. Glover, J. R. Hunt and O. M. Yaghi, Nat. Chem., 2010, 2, 235.

13 Chemspeed Technologies, Augst.

14 N. L. Campbell, R. Clowes, L. K. Ritchie and A. I. Cooper, Chem. Mater., 2009, 21, 204.

15 MS Modeling 4.4, Accelrys Software Inc., 2008.

16 E. K. Sichel, R. E. Miller, M. S. Abrahams and C. J. Buiocchi, Phys. Rev. B: Solid State, 1976, 13, 4607.

17 R. W. Tilford, S. J. Mugavero, P. J. Pellechia and J. J. Lavigne, Adv. Mater., 2008, 20, 2741.

18 K. S. W. Sing, D. H. Everett, R. A. W. Haul, L. Moscou, R. A. Pierotti, J. Rouquerol and T. Siemieniewska, Pure Appl. Chem., 1985, 57, 603.

19 A. R. Leach, Molecular Modelling: Principles and Applications, Prentice Hall, Harlow, England, 2nd edn, 2001.

20 M. Connolly, J. Appl. Crystallogr., 1983, 16, 548.

21 (a) T. Düren, F. Millange, G. Ferey, K. S. Walton and R. Q. Snurr, J. Phys. Chem. C, 2007, 111, 15350; (b) B. Assfur and G. Seifert, Microporous Mesoporous Mater., 2010, 133, 59. 